\title{
Exploring Visual Prominence of Multi-Channel Highlighting in Visualizations
}

\author{
Manuela Waldner \\ TU Wien \\ Austria \\ waldner@cg.tuwien.ac.at
}

\author{
Alexey Karimov \\ CoolIT \\ Korneuburg, Austria \\ Alexey.Karimov@cool-it.at
}

\author{
Eduard Gröller \\ TU Wien \\ Austria \\ groeller@cg.tuwien.ac.at
}

\begin{abstract}
Visualizations make rich use of multiple visual channels so that there are few resources left to make selected focus elements visually distinct from their surrounding context. A large variety of highlighting techniques for visualizations has been presented in the past, but there has been little systematic evaluation of the design space of highlighting. We explore highlighting from the perspective of visual marks and channels - the basic building blocks of visualizations that are directly controlled by visualization designers. We present the results from two experiments, exploring the visual prominence of highlighted marks in scatterplots: First, using luminance as a single highlight channel, we found that visual prominence is mainly determined by the luminance difference between the focus mark and the brightest context mark. The brightness differences between context marks and the overall brightness level have negligible influence. Second, multi-channel highlighting using luminance and blur leads to a good trade-off between highlight effectiveness and aesthetics. From the results, we derive a simple highlight model to balance highlighting across multiple visual channels and focus and context marks, respectively.
\end{abstract}

\section{CCS CONCEPTS}

- Human-centered computing $\rightarrow$ Empirical studies in visualization; • Computing methodologies $\rightarrow$ Perception;

\section{KEYWORDS}

Information visualization, highlighting, focus+context, visual prominence.

\section{ACM Reference Format:}

Manuela Waldner, Alexey Karimov, and Eduard Gröller. 2017. Exploring Visual Prominence of Multi-Channel Highlighting in Visualizations. In SCCG '17: SCCG '17: Spring Conference on Computer Graphics 2017, May 15-17, 2017, Mikulov, Czech Republic. ACM, New York, NY, USA, 10 pages. https://doi.org/10.1145/3154353.3154369

Permission to make digital or hard copies of all or part of this work for personal or classroom use is granted without fee provided that copies are not made or distributed for profit or commercial advantage and that copies bear this notice and the full citation on the first page. Copyrights for components of this work owned by others than the author(s) must be honored. Abstracting with credit is permitted. To copy otherwise, or republish, to post on servers or to redistribute to lists, requires prior specific permission and/or a fee. Request permissions from permissions@acm.org.

SCCG '17, May 15-17, 2017, Mikulov, Czech Republic

(c) 2017 Copyright held by the owner/author(s). Publication rights licensed to the Association for Computing Machinery.

ACM ISBN 978-1-4503-5107-2/17/05 ..\$15.00

https://doi.org/10.1145/3154353.3154369

\section{INTRODUCTION}

Highlighting of visualization elements supports the user when gradually exploring the data, for instance through dynamic queries or brushing and linking across multiple views [Kosara et al. 2003]. It is also a viable component of narrative visualization [Segel and Heer 2010], where known aspects of the data are subsequently presented to the user. The goal of highlighting is to make important data points more visually prominent [Hall et al. 2016]. In terms of low-level graphical processing this means that the visual features (or visual variables [Bertin 1983] or visual channels [Munzner 2014]) of the visualization have to be manipulated in a way that the users' bottom-up attention is effectively attracted by highlighted elements.

Common highlighting techniques in information visualization use the hue [Doleisch et al. 2003; Strobelt et al. 2016], luminance [Bergman et al. 1995; Khan et al. 2005], spatial distortions [Furnas 1986], oscillating movements [Ware and Bobrow 2004], or blur [Kosara et al. 2002] to put more emphasis on the elements in focus. Others add visual cues to the scene, like labels [Gapminder 2016], halo effects [Baer et al. 2009], or visual links connecting items across multiple coordinated views [Steinberger et al. 2011]. Researchers have named these operations "highlighting" [Liang and Huang 2010; Robinson 2011; Strobelt et al. 2016; Ware 2012], "emphasis techniques" [Baer et al. 2009; Hall et al. 2016], "cue-based techniques" [Cockburn et al. 2009], "attention retargeting" [Mateescu and Bajic 2016] or - more general - "focus+context", defined as the "uneven use of graphics resources (space, opacity, color, etc.) for visualization with the purpose to visually discriminate data-parts in focus from their context, i.e., the rest of the data" [Hauser 2006]. In this paper, we use the term "highlighting" to denote the operation to make elements of interest visually distinct. We use the term "focus" for the elements to be highlighted and "context" for the remaining elements.

While highlighting is of general interest for graphical user interfaces [Khan et al. 2005; Zhai et al. 1997], it poses special challenges for the field of visualization. Visualizations generally seek to maximize the amount of information displayed [Haroz and Whitney 2012]. Therefore, multiple visual channels and a large dynamic range within these channels is typically utilized to encode the data. This leaves little capacity for generating visual elements that "pop out" from their surrounding without severely limiting the expressiveness of the context elements (for instance, by shrinking [Furnas 1986] or darkening [Khan et al. 2005] them). Robinson [Robinson 2011] therefore suggests to couple multiple highlighting methods to create a stronger compound highlighting. However, little is known which factors influence the visual prominence of highlighted elements in visualizations, especially for compound highlighting [Hall et al. 2016; Robinson 2011]. 
In this work, our goal is to systematically explore highlighting from the perspective of visual channels, which are directly controlled by visualization designers to create visual encodings. To this end, we present results of two experiments exploring 1) factors that influence the visual prominence of a highlighted element using the luminance channel and 2) the effects of combining the luminance and sharpness channels for highlighting in scatterplots. From the results, we derive a simple multi-channel highlight model.

\section{RELATED WORK}

There are several state-of-the-art reports presenting overviews, design guidelines, and generalizations of highlighting techniques for scientific visualization [Hauser 2006], geovisualization [Robinson 2011], information visualization [Hall et al. 2016; Liang and Huang 2010], and text visualization [Strobelt et al. 2016]. Our goal is also to find a general model for highlighting in visualizations. We approach this challenge by drawing knowledge from the area of human cognitive psychology and visual perception and by adding new insights from experimental findings.

Apart from highlighting techniques that are guided primarily by design recommendations, there have been few examples in prior work that computationally generate highlight effects based on saliency models in images [Hagiwara et al. 2011], videos [Veas et al. 2011], and volume visualizations [Kim and Varshney 2006]. Most of these techniques use a computational saliency model by Itti et al. [Itti et al. 1998] based on "Feature Integration Theory" (FIT) by Treisman and Gelade [Treisman and Gelade 1980]. This saliency model computes a per-pixel saliency map by evaluating center-surround differences for different visual features. Highlighting based on a pixel-based saliency model has several disadvantages when using it for information visualization. First, pixel-based modifications are limited to color channels. Second, most examples above rely on an iterative adjustment procedure to achieve the desired highlight effect [Hagiwara et al. 2011; Lee et al. 2013; Veas et al. 2011]. Finally, efficient pixel-based image modifications require an implementation in a shader [Veas et al. 2011], which severely limits the applicability for information visualization applications.

\section{VISUAL PROMINENCE IN A SINGLE HIGHLIGHT CHANNEL}

We will look at highlighting from the perspective of marks and channels [Munzner 2014]. To generate a highlight effect, the focus mark (i.e., the target) has to be sufficiently dissimilar from the context marks (i.e., the non-targets) in at least one visual channel. There are three properties of a visual channel that could influence the visual prominence of a highlighted focus mark against the set of context marks: the similarity of target and non-targets, the similarity of non-targets, as well as the channel offset (i.e., the lowest value of the non-targets). In psychophysics experiments, it has been shown that search efficiency decreases with increasing target-non-target similarity and with decreasing non-target similarity (similarity theory [Duncan and Humphreys 1989]). In another theory (the relational account of attention [Becker 2010]), the dissimilarity between a target and the non-targets is described by a vector, pointing from the target to the non-targets in feature space. The perceived dissimilarity is quantified by the magnitude of the vector pointing from the target to the closest non-target. When users are given a "search direction" (e.g., "larger" or "brighter"), the attention is guided towards the mark that differs in this direction from all other marks (i.e., the largest or brightest mark). The difference is that, according to similarity theory, the visual prominence of a target decreases when the heterogeneity of the non-targets increases. In contrast, non-target heterogeneity does not have an influence on the visual prominence of the target according to the relational account of attention.

However, findings from classic visual search experiments cannot always be directly applied to information visualization. It has been shown that even small changes to psychophysics experiments, like adding links between dots to simulate node-link diagrams, can have considerable effects on the experimental outcome [Berg et al. 2008]. We therefore performed a visual search experiment to investigate the influence of the three factors (target-non-target similarity, non-target similarity, and offset) on the target's visual prominence using a single highlight channel (CIEL* $\mathrm{a}^{*} \mathrm{~b}^{*}$ luminance) in a classic scatterplot visualization.

\subsection{Stimuli}

We showed a series of d3 [Bostock et al. 2011] scatterplots (based on Gapminder [Bostock 2017b; Gapminder 2016]). The x axis showed the per capita income, and the y axis showed the life expectancy for a single selected year, as in the $\mathrm{d} 3$ online version of Gapminder [Bostock 2017b]. In half of the trials a single highlighted target dot was present, and absent in the other half. Each scatterplot was composed of 150 grayscale dots representing countries, with a 0.5 pixel black border and with 9 pixels radius on white background. In contrast to classic visual search experiments, we also showed the axes, axis labels, and the browser GUI. For each stimulus display, the data set to be displayed was randomly picked out of 209 Gapminder data sets, each showing the income and life expectancy for a selected year, and the target was randomly picked among 150 data points. Targets were always rendered on top, i.e., they were never (partially) occluded by other dots.

\subsection{Apparatus}

We used a BenQ BL2710 27" LCD monitor that was calibrated with an external monitor calibrator using a seven-color sensor. Users were sitting approximately $40 \mathrm{~cm}$ from the display and could move freely. The targets covered around $0.6^{\circ}$ of the visual field, while the entire chart covered approximately $45^{\circ}$ horizontally.

\subsection{Task and Procedure}

At the beginning of the experiment, users received a written instruction to check, as quickly as possible, whether there is a single dot that is brighter than all remaining dots. The users' task was to press the Return key with the right hand as quickly as possible if there was a single dot that was brighter than all other dots, or the space bar with the left hand if no target was present. Before the actual experiment, users could perform unrecorded training sets to get familiar with the controls and the task description. 


\subsection{Design}

We employed a within-subjects design with three independent variables: T-N distance, which is the distance in CIEL ${ }^{*}{ }^{*} b^{*}$ luminance between target and maximum non-target value $(10,20,30), \mathbf{N}$ range, which is the difference between minimum and maximum value of the non-targets $(40,20,10,5)$, and offset, which corresponds to the minimum non-target value $(30,20,10)$, as well as target (present or absent). Example stimuli are shown in Figure 1. Users had to perform two repetitions, resulting in 144 trials. The order of appearance was randomized. Number of dots per trial (150), size of the dots (9 pixel radius), and occlusion (non-occluded targets) were control variables. The spatial location of the target dot and the distribution of dots within the scatterplot were random variables. The dependent variables were response time, measured between stimulus onset and key press, and correctness of the response. Incorrectly answered trials had to be repeated at a later point in the experiment until a correct response was recorded for each condition.

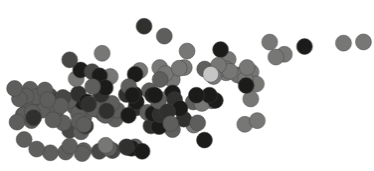

(a)

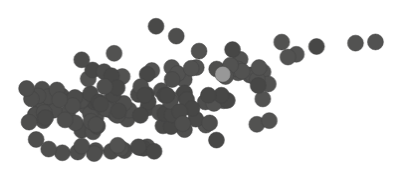

(b)
Figure 1: Sub-set of the stimulus display for T-N distance 30, with $\mathrm{N}$ range 40 and offset $10(\mathrm{a})$, and $\mathrm{N}$ range 5 and off set 30 (b).

\subsection{Subjects}

We tested 8 users (5 males, 3 females, aged 25 to 34), each with self-reported normal or corrected-to-normal vision and naive to the purpose of the experiment. However, some of the users had already participated in a pilot experiment.

\subsection{Results}

Users generally committed few errors in the target-absent trials (3.4\%), but they had $25 \%$ false negative responses for target-present trials. $34 \%$ of targets with T-N distance 10 were missed, but there were only $3 \%$ and $2 \%$ false negatives for T-N distance 20 and 30 , respectively. As usual in visual search experiments, target-absent trials lead to a significantly slower response time $(\bar{r} t=5519 \mathrm{~ms})$ than target-present trials $(\overline{r t}=3581 \mathrm{~ms}, t(7)=5.794, p=.001)$. For further analysis of the visual channel factors, we only considered the target-present trials.

To analyze the influence of the three factors on the response time, we modeled a linear regression using all correct responses of targetpresent trials. Since the obtained data is skewed, we first applied a $\log$-log-transformation on the data and then removed outliers. The linear regression yields a goodness-of-fit of $R^{2}=.531$. T-N-distance is the only significant factor of the regression $(-1.15, p<.001)$, while $\mathrm{N}$-range $(0.04, p=.176)$ and offset $(0.04, p=.348)$ are very small and do not have a significant influence on the model.

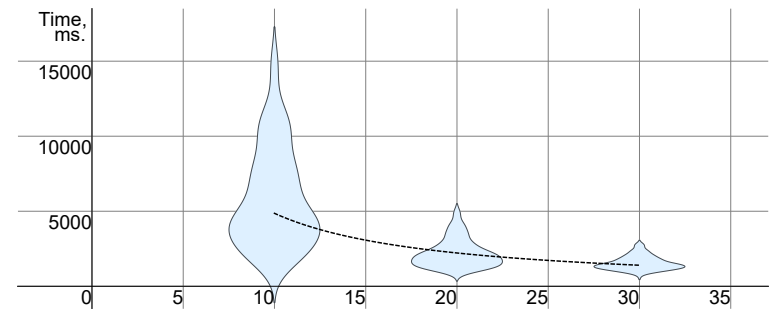

Figure 2: Response time (in $\mathrm{ms}$ ) per T-N distance (CIEL ${ }^{*} \mathbf{a}^{*} \mathbf{b}^{*}$ luminance differences).

A linear regression of log-log-transformed data corresponds to a power regression on the original data. A power regression using T-N-distance as single factor is shown in Figure $2\left(R^{2}=.528\right)$. This means that our response time observations can be explained by Stevens' Power Law [Stevens 1975], which describes the sensation magnitude $\psi$ as a power function of the physical stimulus magnitude $\phi$ :

$$
\psi=k \phi^{\beta},
$$

where the constant $k$ is a scale factor, $\beta$ is the characteristic sensation of the provided stimulus, and the stimulus magnitude $\phi$ is described here by the difference between the target's luminance value and the brightest non-target (i.e., the T-N distance).

\subsection{Discussion}

In our scatterplot visualization, the luminance difference between the target and the brightest non-target was the factor that could best explain the variance in the observed response times. The luminance offset, as well as the variance of the non-target luminances, did not show a significant effect. This observation contradicts the similarity theory [Duncan and Humphreys 1989], which states that both, T-N similarity and N-N similarity contribute to the search efficiency. Consider the example stimuli in Figure 1: according to our results, search for the two targets is equally efficient. Similarity theory, however, would suggest that the target in Figure 1(b) can be found more efficiently. However, one obvious difference between our experiment and classic visual search experiments in vision research is that we had considerable visual noise in our experiment: a white background, black chart decorations, and GUI elements. This, however, is a desirable difference, since we are not interested in explaining the human visual system, but rather guiding the design of an actual application that will be employed in a non-perfect environment [Berg et al. 2008]. One hypothesis therefore is that users are confronted with such a contrast-rich visual environment that the effect of the non-target heterogeneity vanishes.

The other obvious difference to Duncan's and Humphrey's experiments [Duncan and Humphreys 1989] is that users did not have to search for a pre-defined target that was presented to them before each trial. Rather, users had to search for a relatively defined target (i.e., "the brightest"). For such a task, the relational account of attention [Becker 2010] predicts that the visual attention is guided towards the mark that differs in the given search direction from all other marks, irrespective of the non-targets' similarity. In contrast to the experiment series conducted by Duncan and Humphreys [Duncan and Humphreys 1989], where users had to search one 
upright L among $90^{\circ}$ clockwise and / or counter-clockwise rotated non-target Ls, our target only differed in one direction (i.e., increasing luminance) from the non-targets in the highlight channel. One explanation therefore could be that users only investigated the brightest non-targets and could easily ignore the ones that were darker. This means that the relational account of attention [Becker 2010] can better explain the search processes for highlighting than the similarity theory [Duncan and Humphreys 1989].

\section{VISUAL PROMINENCE IN MULTIPLE HIGHLIGHT CHANNELS}

Single highlight channels sometimes do not have the necessary capacities to generate the desired visual prominence. Multi-channel or compound [Robinson 2011] highlighting therefore utilizes the principle of disjunction search: the modification of multiple channels concurrently increases the overall dissimilarity. This means in turn that, given a desired overall visual prominence, the modifications within individual channels can be reduced. It is not clear, however, how much individual channels contribute to the compound visual prominence of the highlighted mark.

In vision research, the dissimilarity of two items $x$ and $y$ across $w$ channels is commonly described by the Minkowski metric [Garner 2014]:

$$
\mathrm{d}(x, y)=\left(\sum_{j=1}^{w}\left|x_{j}-y_{j}\right|^{r}\right)^{1 / r} \mid r \geq 1
$$

If the channels are integral, they cannot be judged independently (like color components). In this case, $r=2$, i.e., the dissimilarity is described by the Euclidean distance. If the channels are separable, like color and group location, $r=1$, which corresponds to the cityblock distance. When combining integral and separable channels, $r$ is between 1 and 2, or different values for $r$ for the various sub-pairs are used [Johannesson 2001].

Based on this metric, we explored the interplay between two highlight channels: luminance $\left(\mathrm{L}^{*}\right)$, as used, for instance, for spotlight highlighting [Khan et al. 2005], and blur / sharpness (s), as used for semantic depth of field highlighting [Kosara et al. 2002], as well as the compound highlighting using both channels ( $\left.\mathrm{L}^{*} \mathrm{~s}\right)$. Through a second visual search experiment, our goal was to answer the following research questions:

Q1: Does highlighting in the luminance and sharpness channel lead to similar visual search behavior?

Q2: Which channel combination factor $r$ (Eq. 2) describes the compound visual prominence of the luminance and sharpness channel? Q3: How does multi-channel highlighting influence the aesthetics and perceived "distortions" of the visualization, compared to single-channel highlighting?

We used the same apparatus and similar stimuli, procedure, and design as in the first experiment. However, this experiment was split into two parts: the visual search part and the subjective dissimilarity part.

\subsection{Stimuli}

Gapminder scatterplot dots were assigned five non-target luminances $(90,80,70,60,50)$. In the $L^{*}$ - and $L^{*} s$-condition, the nontargets were darkened, and in the s- and $\mathrm{L}^{*} \mathrm{~s}$-condition, the nontargets were blurred. Targets were always unblurred, with luminance 80 (Figure 3). To calibrate the visual prominence of the target in the two highlight channels, we adjusted $k$ from Equation 1 for each channel. The inverse of $k$ can be interpreted as the smallest visual change that can be detected by human observers in the respective visual channel. For the luminance channel, we set the inverse of $k$ to 2.3 ( $c f$., Lee et al. [Lee et al. 2013]). For the sharpness channel, we empirically determined a standard deviation of 0.12 for the blur in pilot runs using the actual study configuration. The sensation characteristics $\beta$ from Equation 1 was set to one for both channels.

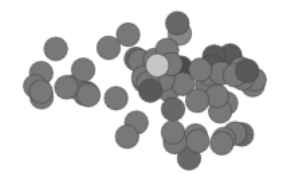

(a) $\mathrm{L}^{*}$

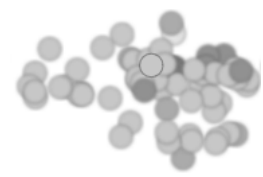

(b) $\mathrm{s}$

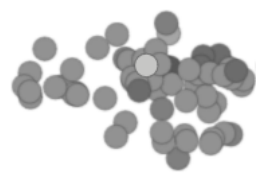

(c) $L^{*} \mathrm{~s}$
Figure 3: Small sub-set of the stimulus display with $\psi=10$.

\subsection{Task and Procedure}

In the visual search part, users were asked to indicate whether there was a highlighted dot, which was either brighter than all other dots, or a single dot that was not blurred, or brighter and not blurred. The trials were blocked by highlight method so that the target description (i.e., "brightest", "not blurred", and "brightest and not blurred") was consistent within a block. In the subjective dissimilarity part, the original scatterplot and a scatterplot containing only the nontargets of the modified scatterplot were shown side-by-side. Users had to report the subjective dissimilarity of the two juxtaposed charts, and the visual appeal of the scatterplot containing the nontargets compared to the original one, on a 5-point Likert scale for each condition. The Likert scales were labeled equal - quite similar - quite dissimilar - very dissimilar - extremely dissimilar and uglynot appealing - neutral - nice - very nice.

\subsection{Design}

We employed a within-subjects design with three independent variables: $\psi$ (Eq. 1), describing the dissimilarity between target and closest non-target value by inverse $k$ steps $(5,10,15,20)$, channel $\left(\mathrm{L}^{*}, \mathrm{~s}, \mathrm{~L}^{*} \mathrm{~s}\right)$, and target (present, absent). In the $\mathrm{L}^{*} \mathrm{~s}$-condition, $\psi$ was simply split up between the channels, which corresponds to a channel combination factor of $r=1$ (Eq. 2). This results in 24 conditions, which were repeated six times in the visual search part, resulting in 144 trials. The trials were blocked by channel with 48 trials. These blocks were counter-balanced per participant. In the subjective dissimilarity part, the 24 conditions were repeated once and presented in random order. As dependent variables, we measured response time and correctness in the visual search part, and 


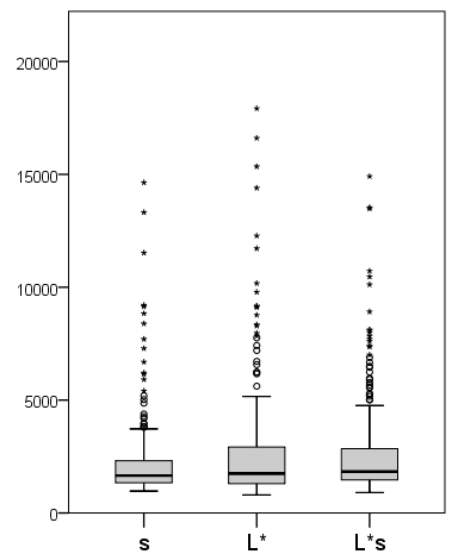

(a) response time $(\mathrm{ms})$

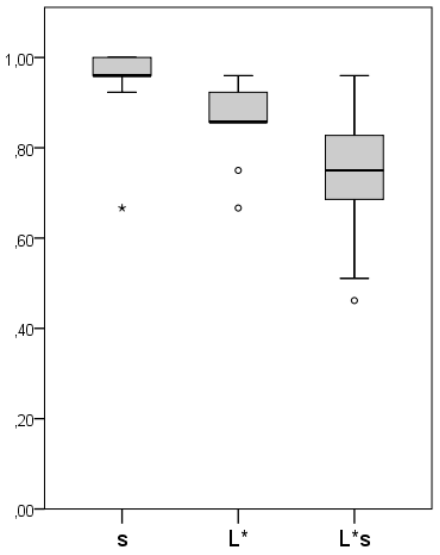

(b) correctness

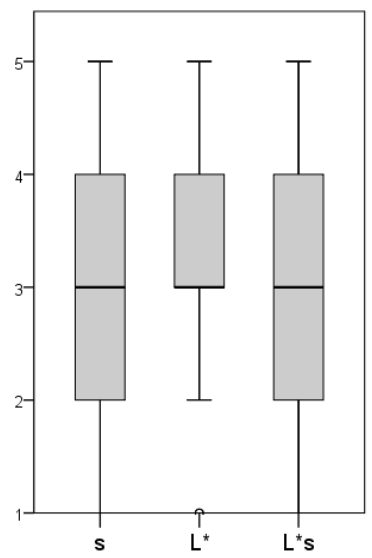

(c) dissimilarity

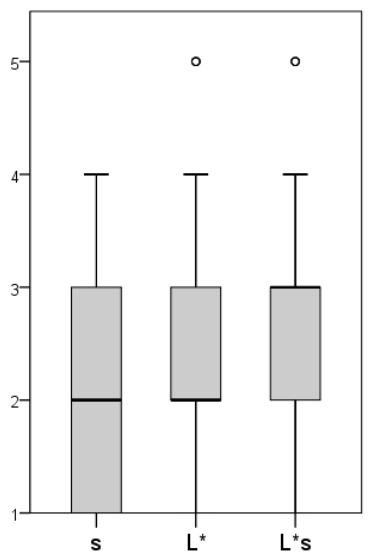

(d) aesthetics

Figure 4: Response times in ms (a), correctness ratio (b), dissimilarity ratings (c), and aesthetics ratings (d) on a 5-point Likert scale, per highlight condition.

subjective dissimilarity and aesthetics in the subjective dissimilarity part.

\subsection{Subjects}

We tested 9 users (7 males, 2 females, aged 22 to 39), each with self-reported normal or corrected-to-normal vision and naive to the purpose of the experiment.

\subsection{Results}

Like in the first experiment, the obtained response times for all correctly answered target-present trails were skewed and therefore log-transformed first. Then, we removed outliers for each level of $\psi$ of the log-transformed response times. However, after these transformations, response times were not normally distributed. To test the difference in visual prominence between the two singlechannel conditions (Q1), we therefore performed a Friedman test, comparing the response times between the three highlight conditions. We found no significant differences between the conditions $\left(\chi^{2}(2)=5.56, p=.062\right)$. On average, the $\mathrm{L}^{*}$-condition led to around $560 \mathrm{~ms}(24 \%)$ slower response times than the s-condition (Figure 4(a)). Also, in the s-condition, users had a lower number of false negative responses $(4.6 \%)$ than in the $\mathrm{L}^{*}$-condition $(9 \%)$, as shown in Figure 4(b). To further explore these differences, we fitted linear regressions for both channels, where the log-transformed response time was the dependent variable, and the log-transformed visual prominence $\psi$ was the independent variable. For the $\mathrm{L}^{*}$-condition, the regression line has a steeper slope, indicating that performance differences between the two channels were primarily caused by trials with low visual prominence $\psi$.

On average, the multi-channel $L^{*} s$-condition also led to around $23 \%$ slower response times than the s-condition. In addition, the highest number of incorrect responses was recorded in the L*scondition (18\%) (see Figure 4). Since we simply split up $\psi$ between the two channels, the lowest possible channel combination factor $r=1$ was used during the experiment - i.e., luminance and sharpness were treated as separable channels. The slightly lower response times and higher error rate indicate that the perceived compound visual prominence is lower than the visual prominence of the single-channel conditions using the same $\psi$. This means that $r$ is presumably larger than 1 . To find a good candidate for the channel combination factor $r(\mathbf{Q 2})$, we tried to find the value of $r$, which optimizes the fit of the response times obtained for the multichannel L*s-condition to the response times of the single-channel $\mathrm{L}^{*}$ - and s-conditions. We therefore modeled one power regression for all samples from the $\mathrm{L}^{*}$ - and s-condition $\left(R^{2}=.450\right)$, and one power regression for the $\mathrm{L}^{*} \mathrm{~s}$-condition $\left(R^{2}=.542\right)$. We then analytically determined the minimum of the inverse goodness-of-fit $\left(1-R^{2}\right)$ of the samples obtained from the $\mathrm{L}^{*}$ - and s-conditions for the L*s-model, as a function of $r$ between 1 and 2. By raising $r$, the intercept of the regression curve gets lower, since the combined sensation magnitude $\psi$ is lowered. We found the best goodness-of-fit for $r=1.22$.

To test the highlight conditions' influence on perceived dissimilarity and aesthetics (Q3), we performed Friedman tests for the dissimilarity and aesthetic scores ( $c f$., Figure 4$)$. We did not find any significant differences for the dissimilarity scores $\left(\chi^{2}(2)=2.80, p=\right.$ .247). For all three conditions, the median score was "quite dissimilar". However, there is a significant effect for the aesthetics scores $\left(\chi^{2}(2)=10.80, p=.005\right)$. Bonferroni-adjusted Wilcoxon-Signed Rank post-hoc comparisons showed that sharpness was rated significantly less appealing than the multi-channel highlight condition $\mathrm{L}^{*} \mathrm{~s}(Z=-2.68, p=.007)$. While the multi-channel condition $\mathrm{L}^{*} \mathrm{~s}$ received mostly neutral responses (average: 2.83 ), the single-channel conditions $\mathrm{L}^{*}$ and $\mathrm{s}$ received more votes for "not appealing".

\subsection{Discussion}

We will discuss the results of this experiment by directly addressing its three research questions:

Q1: Like in the first experiment (Section 3), the response times obtained for luminance and sharpness highlighting are best modeled 
by a power regression and therefore can be explained by Stevens' Power Law (Eq. 1). Differences of response times between the three channel conditions did not reach statistical significance. However, on average, highlighting using sharpness led to more than $500 \mathrm{~ms}$ faster responses and only $50 \%$ of false negative responses compared to luminance-highlighting. It seems that sharpness was more effective especially when the visual prominence of the highlighted target was low. This indicates that the calibration between the two channels can be further improved, for instance by approximating the sensation characteristics $\beta$ (see Eq. 1) for each channel.

Q2: On average, multi-channel highlighting using luminance and sharpness led to slower response times than sharpness-highlighting alone and higher error rates than both single-channel conditions. This is not surprising, given that we used the lowest possible channel combination factor $r$, so that the modifications within the two channels to generate the compound visual prominence was minimal. By increasing the channel combination factor $r$ to 1.22 , the goodness-of-fit of the compound L*s response time model to the response times samples obtained in the single-channel conditions $\mathrm{L}^{*}$ and s could be maximized. This is very close to $r=1.2$, as suggested in previous work [Johannesson 2001], to combine dissimilarities of multiple integral and separable channels into a common dissimilarity model. We therefore conclude that a channel combination factor of $r=1.2$ is a good approximation to model the compound visual prominence generated by multiple highlight channels.

Q3: All three highlight techniques led to comparable perceived dissimilarity with respect to the unmodified scatterplot. Sharpness highlighting, however, received lower ratings for aesthetics than the multi-channel highlight condition. This indicates that the combination of the two channels can provide a good trade-off between highlight effectiveness and aesthetic appeal.

\section{A SIMPLE MULTI-CHANNEL HIGHLIGHT MODEL}

Based on the results of our experiments, we now describe a simple multi-channel highlight model based on the marks' visual channels. We first show how Stevens' Power Law, explaining our experimental results, can be used to generate highlight effects with a desired visual prominence. Given a desired visual prominence of the focus elements $\psi$, we show how this model allows the user to balance the necessary modifications across multiple visual channels and across focus and context marks, respectively. We then exemplify this simple highlight model for other visual channels and visualizations and discuss its limitations and potential future research directions.

\subsection{Focus Enhancement and Context Suppression}

Let $M$ be a set of $n$ graphical marks $M=\left\{m_{i} \mid 1 \leq i \leq n\right\}$, partitioned into focus marks $M_{F}$ and context marks $M_{C}$. The visualization framework controls $1 \leq j \leq w$ continuous visual channels $V=\{$ luminance, sharpness, $\ldots\}$, so that $v_{j}\left(m_{i}\right)=v_{i j}$ describes the value of the $i^{t h}$ mark in the $j^{t h}$ visual channel. Let $\psi$ be the overall desired visual prominence of the focus marks, expressed as perceived dissimilarity of a focus mark to all context marks in units of $\kappa_{j}=1 / k_{j}$. The visual prominence $\psi_{j}$ for a single highlight channel $j$ can be determined by solving Equation 2 so that:

$$
\psi_{j}=\left(\psi^{r} / w\right)^{1 / r},
$$

where $r=1.2$ is a sensible choice to balance the contributions across multiple visual channels (Section 4, [Johannesson 2001]).

In each channel, the marks' values are modified as follows:

$$
v_{i j}^{\prime}= \begin{cases}v_{j}^{f} & \text { if } m_{i} \in M_{F} \\ v_{i j} \cdot \alpha_{j} & \text { if } m_{i} \in M_{C},\end{cases}
$$

where $v_{j}^{f}$ is the focus value of the respective highlight channel, and $\alpha_{j}$ is a scale factor between 0 and 1 that suppresses the context values. The focus channel value $v_{j}^{f}$ and the context scale factor $\alpha_{j}$ are determined by solving the Stevens' Power Law equation (Eq. 1, Section 3), where the stimulus magnitude $\phi_{j}$ is the difference of the focus value $v_{j}^{f}$ to the most prominent non-target in the channel $v_{j}^{\max }:$

$$
v_{j}^{f}=v_{j}^{\max }+\left(\psi_{j} \cdot \kappa_{j}\right)^{1 / \beta_{j}}
$$

and

$$
\alpha_{j}=\frac{v_{j}^{f}-\left(\psi_{j} \cdot \kappa_{j}\right)^{1 / \beta_{j}}}{v_{j}^{\max }} .
$$

We assume that highlight channels are always directed so that $v_{j}^{\max }$ represents the context value with highest visual prominence. For instance, a fully saturated mark will be considered more prominent than a desaturated one, so higher chroma values correspond to higher visual prominence. In other cases, both directions are acceptable. If focus marks are desired to have lower channel values than the context marks, such as lower luminance, the channel values need to be inverted.

By constraining $v_{j}^{f}$, for instance by a maximum luminance value, the modifications of the focus marks (focus enhancement) and the context marks (context suppression) can be balanced. Table 1 illustrates how values within the luminance $\left(\mathrm{L}^{*}\right)$ channel are adjusted for focus enhancement, context suppression, and a combination of these two approaches: In the first example (focus enhancement), no constraints are given, and the desired $\psi=18$ (using $\kappa_{L^{*}}=2.3$ as in Section 4 [Lee et al. 2013]) can be achieved by shifting the luminance of the focus mark within the CIEL* $\mathrm{a}^{*} \mathrm{~b}^{*}$ luminance range $\left(v_{L^{*}}^{f}=99\right)$. Thus, $\alpha_{L^{*}}$ is 1 . In the second example (context suppression), no shifting of the focus value is allowed $\left(v_{L^{*}}^{f}=58\right)$. Therefore, $\alpha_{L^{*}}$ is lower $(\approx 0.3)$, so that context elements get darkened. Finally, to achieve a combination, only half of the desired visual prominence (i.e., $\psi=9$ ) is used for focus enhancement. This way, the modifications are balanced across focus and context marks.

Table 1: Three options to achieve highlighting using luminance.

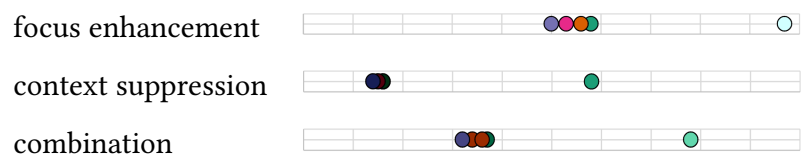




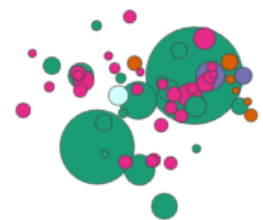

(a) $\mathrm{L}^{*} \mathrm{FE}$

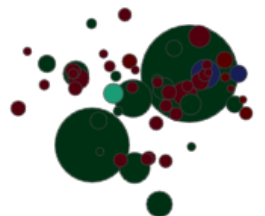

(b) $\mathrm{L}^{*} \mathrm{CS}$

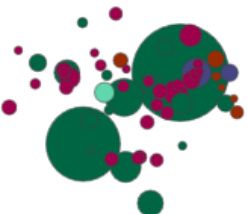

(c) $\mathrm{L}^{*} \mathrm{FE}+\mathrm{CS}$

Figure 5: Comparison of focus enhancement (FE), context suppression (CS), and combined focus enhancement and context suppression $(\mathrm{FE}+\mathrm{CS})$ for luminance $\left(L^{*}\right)$. All examples use the same $\psi$.

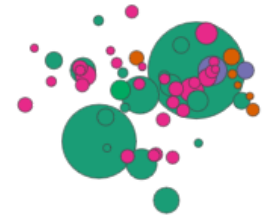

(a) $\mathrm{C}^{*} \mathrm{FE}$

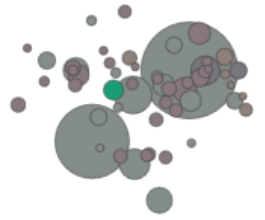

(b) $\mathrm{C}^{*} \mathrm{CS}$

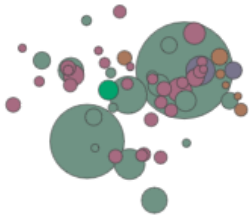

(c) $\mathrm{C}^{*} \mathrm{FE}+\mathrm{CS}$

Figure 6: Comparison of $\mathrm{FE}, \mathrm{CS}$, and $\mathrm{FE}+\mathrm{CS}$ for chroma $\left(\mathrm{C}^{*}\right) \cdot \psi$ is the same for (b) and (c), but limited by the maximum possible chroma value in (a).

In Figure 5, we apply these operations to a Gapminder [Bostock 2017b; Gapminder 2016] chart for the luminance channel. We use the Dark2 color scheme by ColorBrewer [Harrower and Brewer 2003] in our example so that we are able to demonstrate the effect of focus enhancement in the luminance channel with reasonable visual prominence.

\subsection{Examples}

The simple highlight model described above was derived empirically for the luminance and sharpness channel and grayscale scatterplot visualizations. We now exemplify the model on further visual channels and visualizations that have not been investigated in user studies.

5.2.1 Multi-Channel Highlighting in a Scatterplot. In Figure 6, we show focus enhancement and context suppression, as well as combined focus enhancement and context compression for another CIEL ${ }^{*}{ }^{*} b^{*}$ color channel, namely chroma. We use the same settings as described before for Table 1 and Figure 5, respectively. Mind however, that in Figure 6(a), the desired $\psi$ cannot be achieved by focus enhancement alone, since the focus chroma is limited by the maximum possible chroma value. In other words: the saturation of context marks is so high that no sufficient chroma contrast between focus and context can be achieved.

In Figure 7, we show different highlight channel combinations for the same visualization using luminance, chroma, and sharpness. For the sharpness channel, we use $\kappa_{s}=0.12$ (see Section 4).

5.2.2 Choropleth Map. A special challenge in terms of highlighting is geovisualization [Robinson 2011]. Shape, position, and

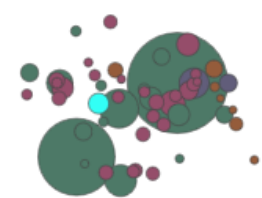

(a) $\mathrm{L}^{*} \mathrm{C}^{*}$

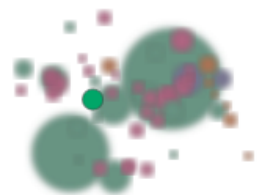

(b) $\mathrm{C}^{*} \mathrm{~s}$

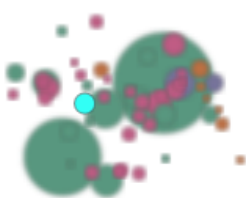

(c) $L^{*} C^{*} s$
Figure 7: Multi-channel highlighting, using combinations of luminance and chroma $\left(L^{*} C^{*}\right)$, chroma and sharpness $\left(C^{*} s\right)$, and luminance, chroma and sharpness $\left(L^{*} C^{*} s\right)$.

size are typically reserved for geographic data encoding. Often, color is used as visual channel to encode quantitative attributes, such as in choropleth maps. In Figure 9(a), we show an example adopted from an existing $\mathrm{d} 3$ choropleth map implementation [Bostock 2017a]. We slightly changed the color coding so that data values are encoded by luminance, while hue and chroma are fairly constant. For highlighting multiple marks, the challenge is to find a second color map for focus marks that stands out from the colors of the context marks. For reference, we also show a naive highlighting approach using red fill color to make the focus elements distinct (Figure $9(\mathrm{~b})$ ). The disadvantage of this approach is that focus marks are not distinguishable, since all color channels are set to the identical focus value.

In this example, we use the simple highlight model to find a visually prominent hue $\left(h^{\circ}\right)$ that stands out from the blue context color. For this purpose, we constrain the hue focus value $v_{h^{\circ}}^{f}$ to the color map's blue hue value plus $\pi$ (see Figure 8 ). Since the chroma of the original choropleth color map is low, we also use the chroma channel to make focus elements stand out: In Figure 9(c), both color channels are used for focus enhancement. In Figure 9(d), the chroma of the focus marks is enhanced, while the chroma of the context marks is suppressed.

Figure 8: Hue ramp used for picking a visually prominent focus color.

5.2.3 Dynamic Queries in a Bubble Chart. Highlighting is also used in combination with dynamic queries to show the result set of the query. Often, queries do not deliver binary results, but assign a continuous degree-of-interest (doi) to particular elements [Furnas 1986]. One advantage of the simple highlight model is that the visual prominence of focus marks can be continuously controlled.

We applied the model for dynamic queries on a d3 BubbleChart [Bostock 2016] (Figure 10(a)) to highlight text query results. Similarity between the user's search string and each mark's text label was determined as Jaccard index of the strings' character threegrams. As this Jaccard index returns a value between zero and one for each mark $i$, we directly use it as scale factor $\operatorname{doi}_{i} \in[0,1]$ for each marks' visual prominence in channel $j: \psi_{i j}=$ doi $_{i} \cdot \psi_{j}$. Each mark's highlight channel value $v_{i j}$ is then adjusted according to its 


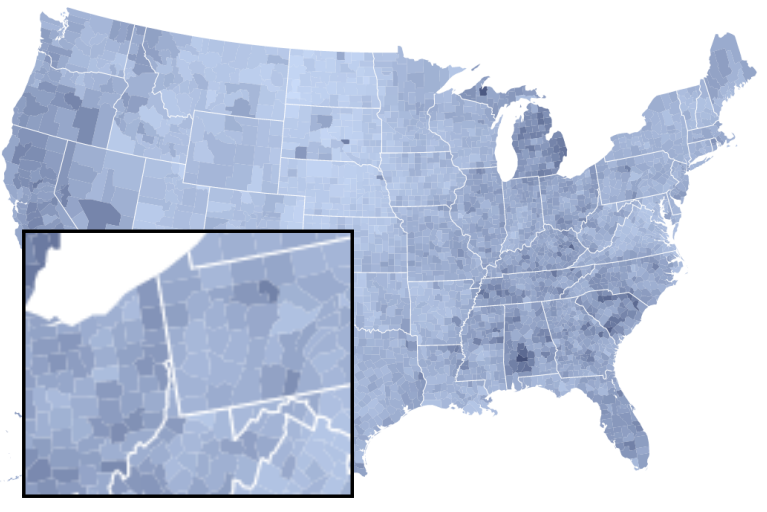

(a)

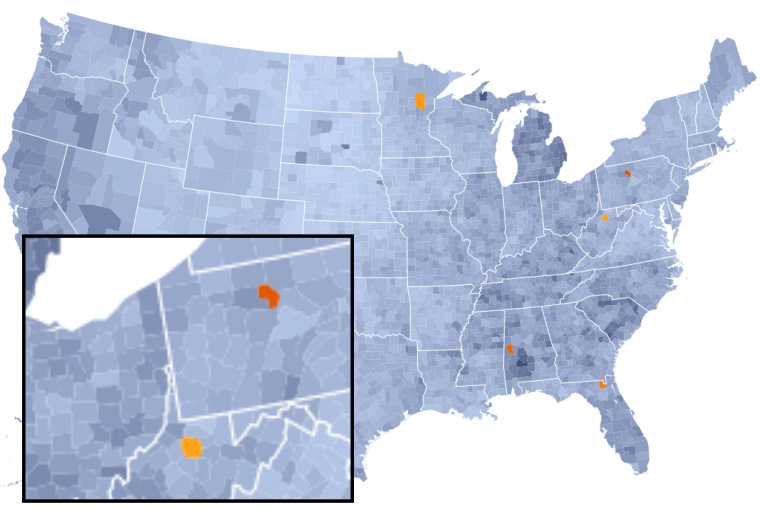

(c)

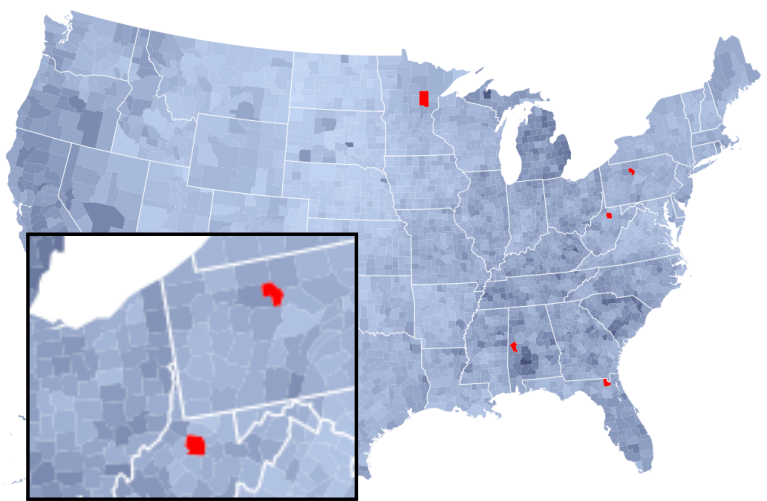

(b)

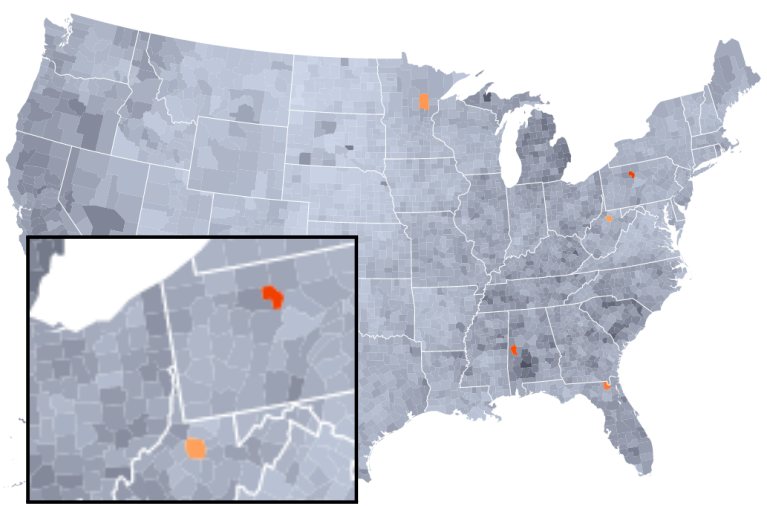

(d)

Figure 9: Choropleth map (adopted from [Bostock 2017a]) (a) with five counties in focus, using red for focus marks (b), using hue and chroma for multi-channel focus enhancement (c), and using hue for focus enhancement and combined focus enhancement and context suppression in the chroma channel (d). Mind how the luminance values encoding the data are preserved in (c) and (d). Manually added insets show close-ups around the two rightmost focus marks.

highlight strength $\psi_{i j}$ :

$$
v_{i j}^{\prime}= \begin{cases}v_{j}^{\max } \cdot \alpha_{j}+\left(\psi_{i j} \cdot \kappa_{j}\right)^{1 / \beta_{j}} & \text { if } \mathrm{doi}_{i}>0 \\ v_{i j} \cdot \alpha_{j} & \text { if } \operatorname{doi}_{i}=0\end{cases}
$$

In Figure 10(b), we show the query results for the search string "data". In this example, the visual channels luminance, chroma, and sharpness are used to encode the text similarity. Through balancing the highlighting across multiple channels, different degrees of interest can be indicated, while still being able to observe clusters encoded by color for both, the elements in focus and in the context.

\subsection{Discussion}

We showed several examples how the simple highlight model could be used to make focus marks visually prominent by only taking the marks' visual channels into account. This is a more formal approach than following design guidelines, and is simple enough to be implemented in any information visualization system, like SVG-based d3 [Bostock et al. 2011].
Our experiments were a step towards exploring the design space of highlighting from the perspective of visual channels. By now, the derived model only considers the marks' values in the highlight channels. Further experimentation is needed to explore the interferences caused by visual channels used for data encoding, such as the size of the marks. Also, the spatial arrangement of marks is likely to have an influence on the focus marks' visual prominence, which could (partially) explain the observed variance in our model.

The examples shown above all perform contrast adjustments in multiple visual channels to generate a highlight effect with a given strength. The same principle could be applied to artificially added visual cues, such as arrows, halos, or visual links. Visual cues do not modify the visualization marks themselves. However, by analyzing the visual channels of the marks in a visualization, we can obtain discriminating channel values for visual cues, such as a prominent hue. To find visually prominent channel values for visual cues, Equation 5 can be used. 


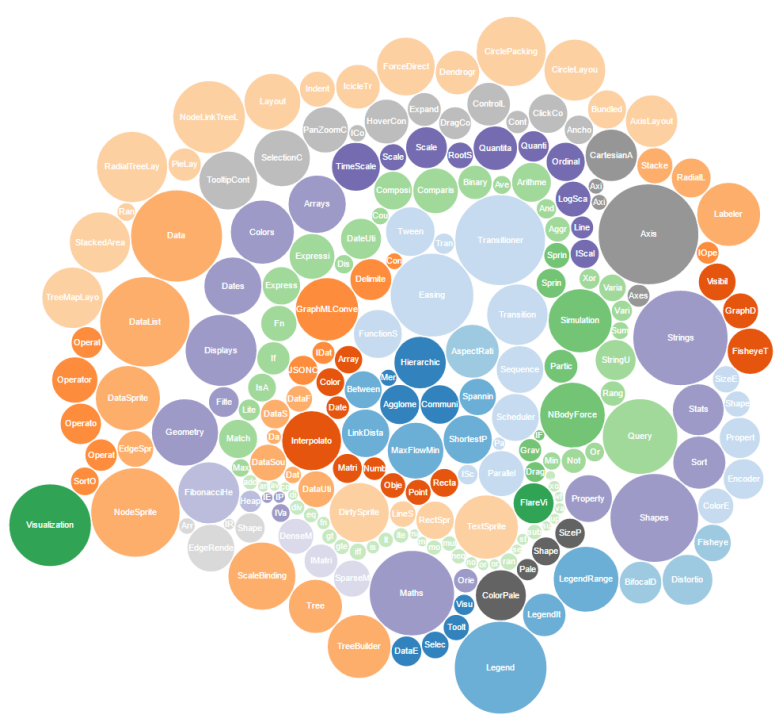

(a) original

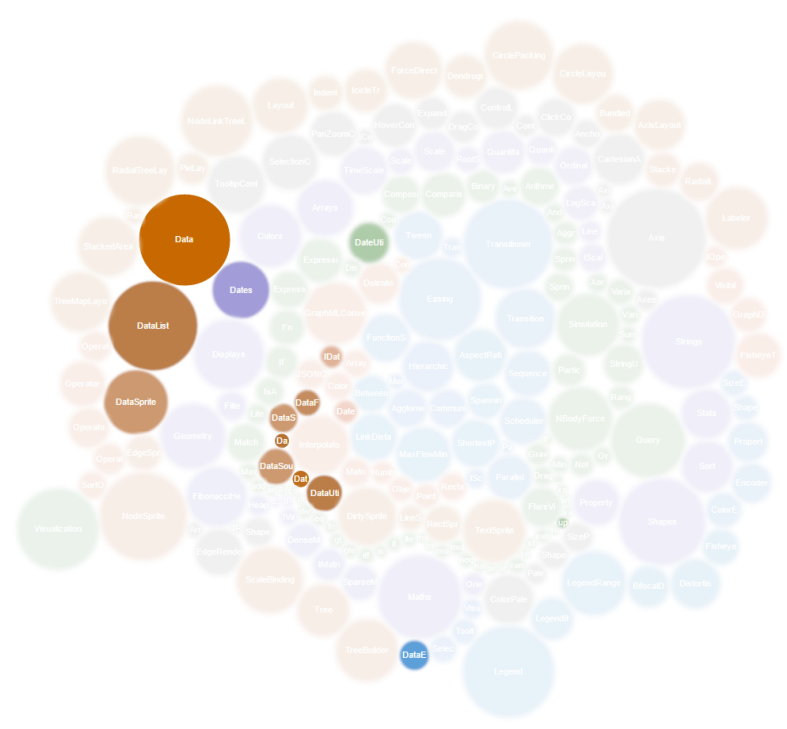

(b) multi-channel highlighting of query result for "data"

Figure 10: d3 BubbleChart [Bostock 2016] (a) with query results for "data" in focus (b), using the highlight channels luminance, chroma, and sharpness. The query returns 15 matches with varying degree of text similarity.

\section{CONCLUSION}

In this paper, we derived a simple model that quantifies the visual prominence of a focus mark against the context marks in visualizations, from two visual search experiments. We used this model to compute multi-channel highlight effects to reach a desired overall visual prominence. This was a step towards systematically exploring the design space of highlighting in visualizations from a visual channel perspective. However, the model can be extended in several ways to make it more accurate, such as by taking the visual channels used for data encoding and spatial proximities into account. Further investigations of highlighting in other visual channels and using different types of visualizations are required for extending the model.

\section{ACKNOWLEDGMENTS}

The authors thank all participants of our experiments. This research has been financed through the Austrian Science Fund FWF (project T 752-N30) and the Vienna Science and Technology Fund WWTF (project VRG11-010).

\section{REFERENCES}

Ra Baer, Friederike Adler, Daniel Lenz, and Bernhard Preim. 2009. Perception-based Evaluation of Emphasis Techniques Used in 3D Medical Visualization. In 3D Medical Visualization. In: Vision, Modeling, and Visualization Workshop. 295-304.

Stefanie I. Becker. 2010. The role of target-distractor relationships in guiding attention and the eyes in visual search. Fournal of Experimental Psychology: General 139, 2 (2010), 247-265. https://doi.org/10.1037/a0018808

Ronald Van den Berg, Frans W. Cornelissen, and Jos B. T. M. Roerdink. 2008. Perceptual Dependencies in Information Visualization Assessed by Complex Visual Search. ACM Transactions on Applied Perception (TAP) 4, 4 (Feb. 2008), 3:1-3:21. https: //doi.org/10.1145/1278760.1278763

L. D. Bergman, B. E. Rogowitz, and L. A. Treinish. 1995. A Rule-based Tool for Assisting Colormap Selection. In Proceedings of the 6th Conference on Visualization '95. IEEE Computer Society, 118-125.
Jacques Bertin. 1983. Semiology of Graphics: Diagrams, Networks, Maps. University of Wisconsin Press.

Mike Bostock. 2016. Bubble Chart. http://bl.ocks.org/mbostock/4063269 (March 2016). Mike Bostock. 2017a. Choropleth. http://bl.ocks.org/mbostock/4060606 (2017).

Mike Bostock. 2017b. The Wealth \& Health of Nations. https://bost.ocks.org/mike/nations/ (2017).

M. Bostock, V. Ogievetsky, and J. Heer. 2011. D3: Data-Driven Documents. IEEE Transactions on Visualization and Computer Graphics 17, 12 (Dec. 2011), 2301-2309. https://doi.org/10.1109/TVCG.2011.185

Andy Cockburn, Amy Karlson, and Benjamin B. Bederson. 2009. A review of overview+detail, zooming, and focus+context interfaces. ACM Computing Surveys (CSUR) 41, 1 (Jan. 2009), 2:1-2:31. https://doi.org/10.1145/1456650.1456652

Helmut Doleisch, Martin Gasser, and Helwig Hauser. 2003. Interactive feature specification for focus+context visualization of complex simulation data. In Proceedings of the symposium on Data visualisation 2003. Eurographics Association, 239-248.

John Duncan and Glyn W. Humphreys. 1989. Visual search and stimulus similarity. Psychological Review 96, 3 (1989), 433-458. https://doi.org/10.1037/0033-295X.96.3. 433

G. W. Furnas. 1986. Generalized fisheye views. In Proceedings of the SIGCHI conference on Human factors in computing systems. ACM, 16-23. https://doi.org/10.1145/22627. 22342

Gapminder. 2016. Gapminder: Unveiling the beauty of statistics for a fact based world view. http://www.gapminder.org/ (2016).

W. R. Garner. 2014. The Processing of Information and Structure. Psychology Press

Aiko Hagiwara, Akihiro Sugimoto, and Kazuhiko Kawamoto. 2011. Saliency-based Image Editing for Guiding Visual Attention. In Proceedings of the 1st International Workshop on Pervasive Eye Tracking and Mobile Eye-based Interaction. ACM, 43-48. https://doi.org/10.1145/2029956.2029968

K. Wm. Hall, C. Perin, P. G. Kusalik, C. Gutwin, and S. Carpendale. 2016. Formalizing Emphasis in Information Visualization. Computer Graphics Forum 35, 3 (June 2016), 717-737. https://doi.org/10.1111/cgf.12936

S. Haroz and D. Whitney. 2012. How Capacity Limits of Attention Influence Information Visualization Effectiveness. IEEE Transactions on Visualization and Computer Graphics 18, 12 (Dec. 2012), 2402-2410. https://doi.org/10.1109/TVCG.2012.233

Mark Harrower and Cynthia A. Brewer. 2003. ColorBrewer.org: An Online Tool for Selecting Colour Schemes for Maps. The Cartographic fournal 40, 1 (June 2003), 27-37. https://doi.org/10.1179/000870403235002042

Helwig Hauser. 2006. Generalizing Focus+Context Visualization. In Scientific Visualization: The Visual Extraction of Knowledge from Data. Springer Berlin Heidelberg, 305-327.

L. Itti, C. Koch, and E. Niebur. 1998. A model of saliency-based visual attention for rapid scene analysis. IEEE Transactions on Pattern Analysis and Machine Intelligence 
20, 11 (1998), 1254-1259. https://doi.org/10.1109/34.730558

Mikael Johannesson. 2001. Combining Integral and Separable Subspaces. In TwentyThird Annual Conference of the Cognitive Science Society. Psychology Press, 447-452.

Azam Khan, Justin Matejka, George Fitzmaurice, and Gordon Kurtenbach. 2005 Spotlight: Directing Users' Attention on Large Displays. In Proceedings of the SIGCHI Conference on Human Factors in Computing Systems. ACM, 791-798. https://doi.org/10.1145/1054972.1055082

Youngmin Kim and A. Varshney. 2006. Saliency-guided Enhancement for Volume Visualization. IEEE Transactions on Visualization and Computer Graphics 12, 5 (Sept. 2006), 925-932. https://doi.org/10.1109/TVCG.2006.174

Robert Kosara, Helwig Hauser, and Donna Gresh. 2003. An Interaction View on Information Visualization. In State-of-the-Art Report. Proceedings of EUROGRAPHICS (2003). 123-137.

R. Kosara, S. Miksch, and H. Hauser. 2002. Focus+context taken literally. IEEE Computer Graphics and Applications 22, 1 (Jan. 2002), 22-29. https://doi.org/10.1109/38.974515

Sungkil Lee, M. Sips, and H.-P. Seidel. 2013. Perceptually Driven Visibility Optimization for Categorical Data Visualization. IEEE Transactions on Visualization and Computer Graphics 19, 10 (Oct. 2013), 1746-1757. https://doi.org/10.1109/TVCG.2012.315

Jie Liang and Mao Lin Huang. 2010. Highlighting in Information Visualization: A Survey. In 14th International Conference Information Visualisation (IV) 2010. 79-85. https://doi.org/10.1109/IV.2010.21

V. A. Mateescu and I. V. Bajic. 2016. Visual Attention Retargeting. IEEE MultiMedia 23, 1 (Jan. 2016), 82-91. https://doi.org/10.1109/MMUL.2015.59

Tamara Munzner. 2014. Visualization Analysis and Design. A K Peters/CRC Press.

Anthony C. Robinson. 2011. Highlighting in Geovisualization. Cartography and Geographic Information Science 38, 4 (Jan. 2011), 373-383. https://doi.org/10.1559/
15230406384373

E. Segel and J. Heer. 2010. Narrative Visualization: Telling Stories with Data. IEEE Transactions on Visualization and Computer Graphics 16, 6 (2010), 1139-1148. https: //doi.org/10.1109/TVCG.2010.179

Markus Steinberger, Manuela Waldner, Alexander Lex, Marc Streit, and Dieter Schmalstieg. 2011. Context-Preserving Visual Links. IEEE Transactions on Visualization and Computer Graphics 17, 12 (2011), 2249-2258. https://doi.org/10.1109/TVCG.2011.183

Stanley Smith Stevens. 1975. Psychophysics. Transaction Publishers.

H. Strobelt, D. Oelke, Bum Chul Kwon, T. Schreck, and H. Pfister. 2016. Guidelines for Effective Usage of Text Highlighting Techniques. IEEE Transactions on Visualization and Computer Graphics 22, 1 (Jan. 2016), 489-498. https://doi.org/10.1109/TVCG. 2015.2467759

Anne M. Treisman and Garry Gelade. 1980. A feature-integration theory of attention. Cognitive Psychology 12, 1 (Jan. 1980), 97-136. https://doi.org/10.1016/0010-0285(80) 90005-5

Eduardo E. Veas, Erick Mendez, Steven K. Feiner, and Dieter Schmalstieg. 2011. Directing attention and influencing memory with visual saliency modulation. In Proceedings of the SIGCHI Conference on Human Factors in Computing Systems. ACM, 1471-1480. https://doi.org/10.1145/1978942.1979158

Colin Ware. 2012. Information Visualization: Perception for Design. Elsevier.

Colin Ware and Robert Bobrow. 2004. Motion to Support Rapid Interactive Queries on Node-link Diagrams. ACM Transactions on Applied Perception (TAP) 1, 1 (July 2004), 3-18. https://doi.org/10.1145/1008722.1008724

Shumin Zhai, Julie Wright, Ted Selker, and Sabra-Anne Kelin. 1997. Graphical Means of Directing Users' Attention in the Visual Interface. In Human-Computer Interaction INTERACT '97. Springer US, 59-66. 